\title{
The clinical application of genetic testing in type 2 diabetes: a patient and physician survey
}

\author{
R. W. Grant • M. Hivert • J. C. Pandiscio • J. C. Florez • \\ D. M. Nathan - J. B. Meigs
}

Received: 4 June 2009 / Accepted: 30 July 2009 /Published online: 2 September 2009

(C) Springer-Verlag 2009

\begin{abstract}
Aims/hypothesis Advances in type 2 diabetes genetics have raised hopes that genetic testing will improve disease prediction, prevention and treatment. Little is known about current physician and patient views regarding type 2 diabetes genetic testing. We hypothesised that physician and patient views would differ regarding the impact of genetic testing on motivation and adherence.

Methods We surveyed a nationally representative sample of US primary care physicians and endocrinologists $(n=304)$, a random sample of non-diabetic primary care patients $(n=$ $152)$ and patients enrolled in a diabetes pharmacogenetics study $(n=89)$.

Results Physicians and patients favoured genetic testing for diabetes risk prediction ( $79 \%$ of physicians vs $80 \%$ of nondiabetic patients would be somewhat/very likely to order/ request testing, $p=0.7)$. More patients than physicians $(71 \%$ vs $23 \%, p<0.01$ ) indicated that a 'high risk' result would be very likely to improve motivation to adopt preventive
\end{abstract}

R. W. Grant $(\varangle) \cdot$ M. Hivert · J. C. Pandiscio - J. B. Meigs Division of General Medicine, Massachusetts General Hospital, 50 Staniford St, 9th floor, Boston, MA 02114, USA

e-mail: Rgrant@partners.org

J. C. Florez • D. M. Nathan

Diabetes Research Center (Diabetes Unit),

Massachusetts General Hospital,

Boston, MA, USA

R. W. Grant · J. C. Florez · D. M. Nathan · J. B. Meigs

Harvard Medical School,

Boston, MA, USA

J. C. Florez

Broad Institute of Harvard and Massachusetts

Institute of Technology,

Cambridge, MA, USA lifestyle changes. Patients favoured genetic testing to guide therapy ( $78 \%$ of patients vs $48 \%$ of physicians very likely to request/recommend testing, $p<0.01$ ) and reported that genetic testing would make them 'much more motivated' to adhere to medications ( $72 \%$ vs $18 \%$ of physicians, $p<$ 0.01). Many physicians (39\%) would be somewhat/very likely to order genetic testing before published evidence of clinical efficacy.

Conclusions/interpretation Despite the paucity of current data, physicians and patients reported high expectations that genetic testing would improve patient motivation to adopt key behaviours for the prevention or control of type 2 diabetes. This suggests the testable hypothesis that 'genetic' risk information might have greater value to motivate behaviour change compared with standard risk information.

Keywords Diabetes prediction - Genetic risk .

Genetic testing $\cdot$ Medication adherence $\cdot$ Patient motivation . Type 2 diabetes

\section{Abbreviation \\ FDA Federal Drug Administration}

\section{Introduction}

The rapidly increasing pace of genetic discovery in type 2 diabetes has led to the identification of nearly two dozen genetic loci associated with an increased risk for developing diabetes $[1,2]$. Building on these advances, researchers are gaining insight into the genetic determinants of response to medical therapy ('pharmacogenomics') [3, 4], the propensity to develop diabetes-related complications [5] and mediators of gene-environment interactions [6-8]. 
Progress in type 2 diabetes genetics has implications not only for our understanding of underlying diabetes pathophysiology but also for how we manage patients with the disease [9]. To justify the potential risk and expense, clinical genetic testing must translate into more effective diabetes care. The potential benefit of type 2 diabetes genetic testing to tailor individual patient management is based on several assumptions: (1) test results will motivate patients with pre-diabetes to adhere to preventive strategies; (2) glycaemic control and risk factor reduction guided by genetic information will achieve management goals more effectively than current approaches; and (3) test results will motivate patients with diabetes to adhere to treatment more effectively. As the science of genetic discovery advances, clinical trials will be needed to provide clinical evidence to support these assumptions.

Although the promise of genome-based 'personalised medicine' has yet to be realised, commercial companies are already offering genome-wide genetic profiling that includes information related to diabetes risk [10]. Given the rapid spread of direct-to-consumer genetic profiling, professional societies will be increasingly called upon to establish standards for the appropriate implementation of new genetic tests [11]. As a first step towards this goal, we surveyed both physicians (primary care and endocrinologists) and patients (with and without diabetes) to assess current views about type 2 diabetes genetic testing related to diabetes prediction, lifestyle adherence and medication management. We tested the hypothesis that physicians would have a more favourable view of genetic testing benefits than patients. This survey was conducted before the publication of recent high-profile reports documenting the marginal improvement in predictive ability of genetic markers above standard clinical risk factors $[12,13]$.

\section{Methods}

\section{Survey development}

We developed a survey for physicians and patients based on a conceptual model of how genetic information might be applied in the clinical setting (Table 1). The three major clinical applications of type 2 diabetes genetic testing in this framework include: (1) prediction of future diabetes risk; (2) elucidation of gene-environment interactions; and
(3) tailoring of medical therapy. As depicted in Table 1, results of genetic testing in these domains have specific clinical implications for physicians (risk assessment, lifestyle modification counselling and medication prescribing decisions) and for patients (risk perception, motivation to adopt lifestyle changes and medication adherence).

Using this framework, we developed novel survey items through a process of expert review and individual physician and patient focus groups. Survey responses were modelled after other validated instruments with a five-level response scale. Survey questions assessed general views about genetic testing and included diabetes-specific questions in the following three domains: (1) testing for risk prediction; (2) testing to motivate behaviour change; and (3) testing to guide medication prescription. Questions for physicians were framed to assess whether physicians would recommend testing and how they expected the results to influence their patients' behaviour, whereas the corresponding questions for patients were framed to assess whether patients would request testing and how they expected the results to influence their own behaviour.

\section{Survey administration—physicians}

Using a commercial database company (MMS Inc., Wood Dale, IL, USA), we identified all clinically active physicians listed in the American Medical Association database in the following four specialties: diabetes, endocrinology, internal medicine and family practice. These physician names were linked to a separate proprietary database of corresponding email addresses. From this population of 72,753 physicians (1,835 diabetes/endocrine, 36,610 internal medicine and 34,308 family practice), we randomly selected 1,500 diabetes/endocrine physicians and 1,500 internal medicine/family practice physicians to receive the survey via email. The first survey was sent in May 2008. A second survey sent 1 month later to eligible physicians who did not initially respond included a $\$ 10$ gift card incentive.

We delivered 2,968 emails and received 320 unique survey responses (response rate 11\%). This response rate is consistent with email-based surveys [14], but raised the concern that respondents may have had a greater interest in genetic-related questions than did non-respondents. To assess for this potential response bias, we contacted a random sample of non-responders ( $n=40,12 \%$ of response cohort size) to ask three questions related to clinical experience and general

Table 1 Conceptual framework for genetic testing in type 2 diabetes

\begin{tabular}{lll}
\hline Domain of genetic information & Application to physician & Application to patient \\
\hline Predicting future diabetes risk & Risk assessment & Risk perception \\
Gene $\times$ environment interactions & Lifestyle modification counselling & Motivation to adopt lifestyle modifications \\
Genetically tailored drug therapy & Medication prescribing decisions & Medication adherence \\
\hline
\end{tabular}


enthusiasm about genetic testing. There were no significant differences between responders vs non-responders in patient panel sizes $(81.5 \%$ vs $85.0 \%$ with $>100$ type 2 diabetes patients, $p=0.8$ ), favourable opinion about genetic testing in general $(84.4 \%$ vs $80.0 \%, p=0.2)$ or likelihood of recommending a 'whole genome' test to a patient if approved by the Federal Drug Administration (FDA) (74.0\% vs 72.5\%, $p=0.8$ ).

\section{Patient surveys}

Survey administration-primary care patients without diabetes Patients with a wide range of risk for developing diabetes were recruited from a primary care clinic affiliated with Massachusetts General Hospital to take part in a brief study designed to validate surrogate measures of metabolic syndrome. Patients with diabetes or cardiovascular disease were excluded. In addition to an anthropomorphic exam and blood draw, patients completed a survey about health behaviours and risk perception that included a subset of questions concerning genetic testing in general and for predicting type 2 diabetes. We contacted 243 patients by phone or mail, of whom 165 verbally agreed to participate $(68 \%) ; 154$ came to the visit and signed consent $(63 \%$ overall enrolment rate). Two patients did not complete the survey, leaving 152 analysed surveys (99\% completion rate).

Survey administration-patients enrolled in diabetes pharmacogenetics study Based on our review of the literature and initial pilot testing, we concluded that most patients in the general population do not have an accurate working knowledge of the concepts underlying pharmacogenetics $[15,16]$ and would therefore have difficulty responding to our survey questions in this area. For this study, therefore, we chose to survey patients with enough basic knowledge in order to obtain reasonably informed answers regarding their views of pharmacogenetic testing in type 2 diabetes. We identified a cohort of patients specifically recruited to a clinical study designed to assess response to metformin and glipizide based on genetic profile [17]. Study participants were recruited based on diagnosis of early diabetes, pre-diabetes or high risk for diabetes (diet-treated type 2 diabetes, abnormal fasting glucose, obesity, a positive family history or a personal history of gestational diabetes or polycystic ovary syndrome) and were informed of the potential relationship between genetic polymorphisms and response to diabetes-related medical therapy. These respondents - while not representative of the general populationcan be considered as representative of the 'early adopters' who would be likely to be in the first wave of patients asking for pharmacogenetic testing when it became available [18]. Seventy-two of the 86 enrolled study participants agreed to complete our survey during their study visit (84\%). We also contacted, by phone and by mail, patients who were initially recruited to the pharmacogenetics study but who were then identified as ineligible due either to non-white race/ethnicity or to already being on the study drug. Eighteen out of 25 patients contacted (72\%) completed surveys. Thus, the overall response rate was $81 \%(90 / 111)$.

\section{Statistical methods}

Our primary analysis contrasted responses of physicians vs patients. For pre-diabetes risk assessment and prevention questions we limited patient responses to the group without diabetes, whereas for pharmacogenetics questions we focused specifically on patients already diagnosed with diabetes or prediabetes. In an a priori planned secondary analysis, we also specifically contrasted responses by primary care vs diabetes specialist physicians. Because primary care and diabetes specialists were found to have statistically similar responses to the genetic testing questions, we grouped all physicians when comparing to patient responses. Categorical responses were compared using $\chi^{2}$ tests. Binary variables were created from the five-point response scales by grouping the two most positive (Very or somewhat likely/motivated) vs the three remaining response categories. In an exploratory analysis, we also repeated all analyses after re-grouping the responses as most positive (Very likely/motivated) vs the remaining four categories. All statistical analyses were performed using SPSS (SPSS, Chicago, IL, USA). The Partners Human Research Committee approved the study.

\section{Results}

Survey respondents

Physician respondents $(n=304)$ were experienced $(50 \%$ had 20 years since medical school graduation), tended to see patients in private practice $(62 \%)$ or academic settings (27\%), and were generally confident with diabetes management (97\% of diabetes specialists and $71 \%$ of generalists were 'very confident') (Table 2).

Most patient respondents were non-Hispanic white (96\%). The mean patient age was 55.2 years. The majority ( $82 \%$ ) had some college-level education. There were no significant demographic differences between survey respondents with or without diabetes (Table 3).

Primary care vs diabetes specialist physician responses

Most primary care $(n=129)$ and specialist $(n=175)$ physicians had 'somewhat or very positive' views about diabetes genetic testing. We found no statistically significant differences when comparing physicians based on combining the 'Somewhat' 
Table 2 Physician responder demographics

${ }^{a}$ Comparisons are between generalists and diabetes specialist physicians

DM, type 2 diabetes mellitus

\begin{tabular}{lcccc}
\hline Characteristic & Total (\%) & Generalists (\%) & Diabetes specialists (\%) & $p$ value $^{\mathrm{a}}$ \\
\hline$n$ & 304 & 129 & 175 & \\
Time since medical school (years) & & & & \\
$\quad 20$ & 50.0 & 39.5 & 57.7 & 0.01 \\
Primary clinical practice type & & & & 0.01 \\
Academic/university practice & 26.6 & 19.4 & 32.0 & \\
Community health centre & 7.9 & 10.1 & 6.3 & \\
Private practice & 62.2 & 63.3 & 1.2 & $<0.01$ \\
Other & 3.3 & 6.2 & 90.2 & $<0.01$ \\
$>100$ patients with DM/year & 81.7 & 69.8 & 96.6 & \\
'Very confident' managing DM & 85.5 & 70.5 & & \\
Patient panel characteristics & & & 22.9 & 0.48 \\
$>20 \%$ covered by Medicaid & 29.5 & 38.6 & 62.3 & 0.80 \\
$>20 \%$ racial/ethnic minority & 60.5 & 58.1 & 28.7 & \\
$\quad>20 \%$ non-English primary & 29.4 & 30.2 & & \\
$\quad$ language & & & & \\
\hline
\end{tabular}

and 'Very' response categories. When comparisons were limited to respondents who answered at the extreme of the 5point response scale, however, diabetes specialists had somewhat greater enthusiasm (Table 4). For example, more specialists would definitely order FDA-approved genetic testing to predict type 2 diabetes ( $27 \%$ vs $14 \%$ of generalists, $p<0.01)$ and FDA-approved pharmacogenetic testing to guide therapy ( $54 \%$ vs $40 \%, p=0.02)$. More specialists also reported that a 'high risk' genetic result for developing diabetes would make them much more likely to prescribe metformin $(24 \%$ vs $13 \%, p=0.03)$.

\section{Physician vs patient views on genetic testing}

Physicians had slightly more favourable views regarding genetic testing in general ( $88 \%$ 'somewhat/very positive' opinion) compared with patients (79\% 'somewhat/very positive' opinion, $p<0.01$ ), although this was tempered by a greater concern regarding patient privacy issues $(76 \%$ of physicians vs $49 \%$ of patients 'somewhat/very concerned', $p<0.01$ ) (Table 5). When presented with the idea of 'one simple test to check your (your patients') genetic risks for
100 common diseases', $74 \%$ of physicians and $83 \%$ of patients indicated that they would be 'somewhat/very likely' to either recommend or request such a test $(p=0.02)$.

The proportion of non-diabetic primary care patients who would be very likely to request a genetic test to determine whether they were at high risk for developing diabetes was more than double the proportion of physician respondents who would be very likely to recommend such testing (53\% vs $22 \%, p<0.01)$. Similarly, a greater proportion of patients reported that a 'high risk' result from such testing would make them much more motivated to adopt prescribed lifestyle changes $(71 \%$ vs $23 \%$ of physicians who believed patients would be much more motivated, $p<0.01)$. Only two patients $(1.3 \%)$ reported that receiving a 'low risk' result would make them 'much less motivated', and 29 patients (19.2\%) would be 'somewhat less motivated' to adopt prescribed lifestyle changes.

The proportion of patients with type 2 diabetes or prediabetes who would be very likely to request a genetic test to predict the 'best' diabetes medicine was significantly greater than the proportion of physicians who would be very likely to order such pharmacogenetic testing ( $78 \%$ vs $48 \%, p<0.01$ ).
Table 3 Patient responder demographics

\footnotetext{
${ }^{\text {a }}$ Comparisons are between nonDM primary care patients and DM study patients

DM, type 2 diabetes mellitus
}

\begin{tabular}{lcccc}
\hline Characteristic & Total (\%) & Non-DM patients (\%) & DM study patients (\%) & $p$ value $^{\mathrm{a}}$ \\
\hline$n$ & 241 & 152 & 89 & \\
Age, years (SD) & $55.2(14.4)$ & 53.3 & 49.4 & 0.27 \\
Women & 51.9 & 94.7 & 97.8 & 0.60 \\
White, non-Hispanic & 95.9 & 66.9 & 60.2 & 0.33 \\
Employed (full or part time) & 64.4 & 80.8 & 83.0 & 0.33 \\
Some college education & 81.6 & 152 & 89 & 0.73 \\
\hline
\end{tabular}


Table 4 Comparison of generalists vs specialists physician opinions towards genetic testing $(n=304)$

\begin{tabular}{|c|c|c|c|}
\hline Opinion & $\begin{array}{l}\text { Generalists } \\
(n=129)\end{array}$ & $\begin{array}{l}\text { Diabetes specialists } \\
(n=175)\end{array}$ & $p$ value \\
\hline \multicolumn{4}{|l|}{ General } \\
\hline Very positive opinion of genetic testing & 14.0 & 25.7 & 0.02 \\
\hline Very likely to recommend a 'whole genome' test & 19.4 & 21.7 & 0.67 \\
\hline Very concerned about privacy issues & 31.0 & 37.7 & 0.27 \\
\hline \multicolumn{4}{|l|}{ Pre-diabetes } \\
\hline Very likely to order genetic test to predict risk of developing type $2 \mathrm{DM}$ & 14.0 & 27.4 & 0.01 \\
\hline $\begin{array}{l}\text { Physician believes patients would be 'very much' more motivated to make lifestyle changes } \\
\text { in response to a 'high risk' result }\end{array}$ & 20.9 & 24.6 & 0.49 \\
\hline Very likely to spend more time counselling lifestyle changes in response to a 'high risk' result & 21.9 & 18.9 & 0.56 \\
\hline Very likely to prescribe metformin in response to a 'high risk' result & 13.3 & 24.0 & 0.03 \\
\hline Very likely to more aggressively treat cardiac risk factors in response to a 'high risk' result & 21.9 & 25.3 & 0.59 \\
\hline Very likely to recommend $T C F 7 L 2$ genotype test to pre-diabetic patients & 29.5 & 32.6 & 0.62 \\
\hline \multicolumn{4}{|l|}{ Pharmacogenetics } \\
\hline Very likely to recommend test for diabetes medication profile & 39.5 & 53.7 & 0.02 \\
\hline $\begin{array}{l}\text { Physician believes patients would be 'very much' more motivated to take the medication based } \\
\text { on test result }\end{array}$ & 10.9 & 24.0 & $<0.01$ \\
\hline Very likely to prescribe different medicine than usual based on test result & 39.5 & 42.5 & 0.64 \\
\hline Very likely to order test before clinical trials demonstrate beneficial impact on care & 5.4 & 7.4 & 0.64 \\
\hline
\end{tabular}

Values are \% of physicians responding in the 'Very' category to each listed question

DM, type 2 diabetes mellitus

Most of these patients also reported that a genetic testing approach to guide medication choices would make them 'much more motivated' to adhere to their prescribed medicine ( $72 \%$ vs $18 \%$ of physicians who believed that pharmacogenetic testing would make their patients 'much more motivated' to adhere to medications, $p<0.01$ ).
Patients with some college education had greater enthusiasm for genetic testing in general (42\% with 'very positive' opinion vs $21 \%$ of patients without any college education, $p=$ 0.01). College-educated patients also favoured diabetesrelated genetic testing, although differences were not statistically significant: genetic testing to predict diabetes

Table 5 Comparison of physician and patient responses

\begin{tabular}{|c|c|c|c|c|c|}
\hline Opinion & Physicians & All patients & Non-DM patients & DM study patients & $p$ value \\
\hline$n$ & 304 & 241 & 152 & 89 & \\
\hline \multicolumn{6}{|l|}{ General } \\
\hline Very positive opinion of genetic testing & 20.7 & 38.1 & & & $<0.01$ \\
\hline Very likely to recommend/request a 100 -gene testing panel & 20.7 & 51.9 & & & $<0.01$ \\
\hline Very concerned about privacy issues & 34.9 & 20.9 & & & $<0.01$ \\
\hline \multicolumn{6}{|l|}{ Pre-diabetes } \\
\hline $\begin{array}{l}\text { Very likely to order/request genetic test to predict risk of } \\
\text { developing type } 2 \mathrm{DM}\end{array}$ & 21.7 & & 53.0 & & $<0.01$ \\
\hline $\begin{array}{l}\text { Effect of 'high risk' result on how much more motivated } \\
\text { patients would be to make lifestyle changes }\end{array}$ & 23.0 & & 70.9 & & $<0.01$ \\
\hline \multicolumn{6}{|l|}{ Pharmacogenetics } \\
\hline Very likely to recommend test for diabetes medication profile & 47.7 & & & 78.4 & $<0.01$ \\
\hline $\begin{array}{l}\text { Effect of test result on how much more motivated patients would } \\
\text { be to take the medication }\end{array}$ & 18.4 & & & 71.6 & $<0.01$ \\
\hline
\end{tabular}

${ }^{a}$ Non-DM patients $(n=152)$

${ }^{\mathrm{b}}$ DM study patients $(n=89)$

DM, type 2 diabetes mellitus; DM study patients, participants in a diabetes pharmacogenetics clinical study; Non-DM patients, primary care patients without diabetes 
risk (58\% 'very likely to request testing' vs $50 \%$ without any college education, $p=0.5$ ), and genetic testing to guide therapy $(81 \%$ 'very likely to request pharmacogenetic testing' vs $64 \%$ without any college education, $p=0.18$ ).

\section{Discussion}

We surveyed physicians and patients to ascertain current views regarding the potential clinical impact of type 2 diabetes genetic testing. Both physician and patient survey respondents reported high expectations that genetic test results would significantly impact patient motivation to adopt healthy lifestyle changes and to adhere to prescribed medicines. Moreover, most physicians would order FDAapproved genetic testing to guide their medication choices (48\% 'very likely', 44\% 'likely'). Our results suggest that once available, genetic testing is likely to be widely requested. Rapid adoption of this new technology will undoubtedly add significant cost to the already expensive and relatively inefficient process of diabetes care in the US. Thus, our survey underscores the need to link advances in diabetes genetics with research into the efficacy and cost effectiveness of applying diabetes genetic data in the clinical care setting.

Both physician and patient respondents reported that genetic test results would have a significant impact on motivating behaviour change to adopt a healthier lifestyle. Because healthy lifestyle adoption represents one of the most effective, inexpensive and underachieved modalities of diabetes care and prevention, true improvements in motivation would have significant benefits, potentially justifying costs [19]. However, whether anticipated enthusiasm will translate into actual changes in behaviour remains a critically unanswered question. There is ample reason for scepticism, given that other forms of risk calculation have had limited or no impact on patient behaviour to adopt daily, long-term behavioural changes such as exercise regimens and dietary changes [20, 21]. However, it is certainly possible that there is something uniquely powerful about one's genetic information that will indeed change some patients' behaviour. The converse of the potential benefit of genetic testing is that certain test results could actually decrease motivation. Although patients in our study did not report that being 'low risk' would decrease motivation, this finding also needs to be confirmed in actual clinical studies. Given the very preliminary stage of the science, it is not currently possible to provide accurate, personalised and quantifiable genetic risk information. For the purposes of this survey, therefore, 'high risk' was not explicitly quantified and therefore left to the patient to interpret. In the near future, clinical trials will be needed to specifically investigate the impact of actual genetic test results on patient behaviour and motivation and on physician counselling and prescribing practices.

Both physicians and patients had positive views of genetic testing to guide medication therapy, particularly with regards to medication adherence. As with the anticipated impact on lifestyle change motivation described above, however, the actual impact on medication adherence requires direct evaluation. Embrace of genetic testing to tailor therapy is an approach that contrasts directly with recently published guidelines that recommend sequential drug prescription and dose modification until glycaemic goals are reached [22]. The key question here is whether the cost of a genetic test would justify its use over the current 'trial and error' approach to drug selection. At the heart of this debate is whether the benefit of treatment accrues directly from the amount of $\mathrm{HbA}_{1 \mathrm{c}}$ decline, or whether there are drug-specific benefits beyond $\mathrm{HbA}_{1 \mathrm{c}}$ control. Similarly, such a genotype-guided approach may prove beneficial if the genetic bases of drug-specific deleterious side effects prove to be robust and reproducible.

Another important, though not unexpected finding was that enthusiasm for genetic testing among patients was higher in patients who had more education. This result implies that should genetic testing become available and have proven efficacy, we will have to invest significant resources in raising patients' understanding of the meaning and implications of such a test.

In summary, our results indicate a high baseline state of enthusiasm for the potential roles of diabetes genetic testing to improve clinical care. Moreover, these results suggest the testable hypothesis that genetic risk information might have greater impact on motivating behaviour change compared with standard risk information (e.g. family history, body weight or fasting glucose levels). However, in the absence of data, we must balance genuine enthusiasm for new clinical advances in diabetes genetics with the recognition that we have limited success to date in motivating the majority of patients with type 2 diabetes to successfully and durably maintain significant weight loss and other lifestyle changes. Similarly, although pharmacogenetics offers the promise of tailored drug therapy, the fact remains that despite recent improvements, most patients with type 2 diabetes in the US do not reach American Diabetes Association goals of glycaemic control [23, 24]. As more accurate diabetes genetic testing becomes available, work will be needed to effectively implement such testing into effective diabetes management.

Acknowledgements R. W. Grant is supported by a National Institutes of Health career development award (NIDDK K23 DK067452). This study was funded in part by the Charlton Family Fund.

Duality of interest statement The authors declare that there is no duality of interest associated with this manuscript. 


\section{References}

1. Frayling TM (2007) Genome-wide association studies provide new insights into type 2 diabetes aetiology. Nat Rev Genet 8: 657-662

2. Florez JC (2008) The genetics of type 2 diabetes: a realistic appraisal in 2008. J Clin Endocrinol Metab 93:4633-4642

3. Pearson ER, Donnelly LA, Kimber C et al (2007) Variation in TCF7L2 influences therapeutic response to sulfonylureas: a GoDARTs study. Diabetes 56:2178-2182

4. Feng Y, Mao G, Ren X et al (2008) Ser1369Ala variant in sulfonylurea receptor gene $\mathrm{ABCC} 8$ is associated with antidiabetic efficacy of gliclazide in Chinese type 2 diabetic patients. Diabetes Care 31:1939-1944

5. Pezzolesi MG, Poznik GD, Mychaleckyj JC et al (2009) Genomewide association scan for diabetic nephropathy susceptibility genes in type 1 diabetes. Diabetes 58:1403-1410

6. Florez JC, Jablonski KA, Bayley N, Diabetes Prevention Program Research Group et al (2006) TCF7L2 polymorphisms and progression to diabetes in the Diabetes Prevention Program. N Engl J Med 355:241-250

7. Ludovico O, Pellegrini F, Di Paola R et al (2007) Heterogeneous effect of peroxisome proliferator-activated receptor g2 Ala12 variant on type 2 diabetes risk. Obesity 15:1076-1081

8. Moore AF, Jablonski KA, Mason CC, Diabetes Prevention Program Research Group et al (2009) The association of ENPP1 K121Q with diabetes incidence is abolished by lifestyle modification in the Diabetes Prevention Program. J Clin Endocrinol Metab 94:449-455

9. Grant RW, Moore AF, Florez JC (2009) The genetic architecture of type 2 diabetes: recent progress and clinical implications. Diabetes Care 32:1107-1114

10. Bonetta L (2008) Getting up close and personal with your genome. Cell 133:753-756

11. Burke W, Psaty BM (2007) Personalized medicine in the era of genomics. JAMA 298:1682-1684

12. Meigs JB, Shrader P, Sullivan LM et al (2008) Genotype score in addition to common risk factors for prediction of type 2 diabetes. N Engl J Med 359:2208-2219
13. Lyssenko V, Jonsson A, Almgren P et al (2008) Clinical risk factors, DNA variants, and the development of type 2 diabetes. $\mathrm{N}$ Engl J Med 359:2220-2232

14. Cook C, Heath F, Thompson RL (2000) A meta-analysis of response rates in web- or internet-based surveys. Educ Psychol Meas 60:821-836

15. Morren M, Rijken M, Baanders A, Bensing L (2007) Perceived genetic knowledge, attitudes towards genetic testing, and the relationship between these among patients with a chronic disease. Patient Educ Couns 65:197-204

16. Calsbeek H, Morren M, Bensing J, Rijken M (2007) Knowledge and attitudes towards genetic testing: a two year follow-up study in patients with asthma, diabetes mellitus and cardiovascular disease. J Genet Couns 16:493-504

17. Massachusetts General Hospital Center for Human Genetic Research (2006) SugarMGH. Available from http://www2.mass general.org/chgr/SUGARMGH.htm, accessed 1 June 2009

18. Berwick DM (2003) Disseminating innovations in health care. JAMA 289:1969-1975

19. Herman WH, Hoerger TJ, Brandle M, Diabetes Prevention Program Research Group et al (2005) The cost-effectiveness of lifestyle modification or metformin in preventing type 2 diabetes in adults with impaired glucose tolerance. Ann Intern Med 142:323-332

20. Pijl M, Timmermans DRM, Claassen L et al (2009) Impact of communicating familial risk of diabetes on illness perceptions and self-reported behavioral outcomes: a randomized controlled trial. Diabetes Care 32:597-599

21. Marteau TM, Lerman C (2001) Genetic risk and behavioural change. BMJ 322:1056-1059

22. Nathan DM, Buse JB, Davidson MB et al (2009) Medical management of hyperglycemia in type 2 diabetes: a consensus algorithm for the initiation and adjustment of therapy: a consensus statement of the American Diabetes Association and the European Association for the Study of Diabetes. Diabetologia 52:17-30

23. Hoerger TJ, Segel JE, Gregg EW, Saaddine JB (2008) Is glycemic control improving in U.S. adults? Diabetes Care 31:81-86

24. Engelgau MM, Geiss LS, Saaddine JB et al (2004) The evolving diabetes burden in the United States. Ann Intern Med 140:945950 